\title{
The contribution of discrete transitions to integrated inner shell ion- isation cross sections
}

\author{
David $\operatorname{Rez}\left({ }^{1}\right)$ and Peter $\operatorname{Rez}\left({ }^{2}\right)$ \\ (1) Pennine House, Pennine Drive, London NW2 1PA, England \\ $\left({ }^{2}\right)$ Center for Solid State Science and Department of Physics, Arizona State University, Tempe, AZ \\ 85287-1704, U.S.A.
}

(Received September 25, 1992; accepted November 09, 1992)

\begin{abstract}
Previous calculations of inner shell cross sections used in quantitative microanalysis have neglected transitions to discrete states that appear in spectra as white lines. White line are especially prominent in $\mathrm{L}_{23}$ edges from 1st and 2nd row transition elements and $\mathbf{M}_{45}$ edges in rare earth elements. These discrete features can make a substantial contribution to the integrated cross section, especially for small energy windows less than $50 \mathrm{eV}$. In this paper we present results for 2p-3d transitions for 1 st and 2 nd row transition elements and $3 \mathrm{~d}-4 \mathrm{f}$ transitions for rare earth elements, using Dirac Slater wave functions. The effects of different ionisation states are examined and the results compared to experiment.
\end{abstract}

\section{Introduction.}

Quantitative electron energy loss spectroscopy involves comparing integrated edge intensities with appropriate cross sections, which can either be derived from measurements on standards or calculated from theory [1]. The calculated cross sections consider ejection of the inner shell electron to free electron continuum states and both hydrogenic [2] and Hartree-Slater [3, 4] wave functions have been used to describe the electron wave functions. The transitions to continuum states give rise to the slowly varying edge shape on which fine structures are superimposed. Solid state effects, which give fine structure, are neglected and, if a sufficiently large energy window is chosen it appears that they change the integrated cross section by less than 5\% [5]. In some cases, such as $\mathrm{L}_{23}$ edges in transition metals and $\mathrm{M}_{45}$ edges in rare earth elements, sharp lines appear at the threshold [6]. In an atomic picture these are due to transitions to empty bound $d$ like states for the $L$ edges and empty $f$ states for the $M$ edges of the rare earths. These are neglected in the oscillator strength calculations involving continuum states, though Egerton has attempted an empirical correction in the SIGMAL program [1]. These discrete transitions make a substantial contribution to the integrated intensity especially for small energy windows of less than $50 \mathrm{eV}$. The components of the white lines do not follow the statistical ratio expected from 
the initial state occupation and, when studied at high energy resolution, show a rich fine structure. This has been successfully explained using atomic multiplet theory, sometimes with extra terms in the Hamiltonian for crystal field effects [7-11]. The intensity in the white lines has also been related to the number of empty d states in intermetallic alloys by Pearson et al. [12]. They used the ratio of the white lines to the continuum intensity as a measure of the strength of the white line component and compared this to calculations. However they did not present results on the absolute oscillator strength of the white lines.

In this paper we are concerned with only the total intensity in the white lines and not the fine details of the white line structure or the redistribution of intensity between the two components. Oscillator strengths for $2 p-3 d$ transitions have been published by Manson and Kennedy [13] and Scofield [14]. We present tables of oscillator strengths not only for $2 p-3 d$ transitions for 1 st row transition elements, but also for $2 \mathrm{p}-4 \mathrm{~d}$ transitions for 2 nd row transition elements and $3 \mathrm{~d}-4 \mathrm{f}$ transitions for rare earth elements. These can be used to generate integrated cross sections under the dipole approximation, which can then be added to cross sections for transitions to continuum states. We shall also compare ratios of white line intensities to the integrated continuum contribution using experimental spectra.

\section{Theory.}

The differential cross section in the first Born approximation for transitions between initial state $\left|n_{1}\right|>$ and final state $<\varepsilon l^{\prime} \mid$ is given by

$$
\frac{\mathrm{d}^{2} \sigma}{\mathrm{d} E \mathrm{~d} \Omega}=\left(\frac{4 \gamma^{2}}{a_{0}^{2} q^{4}}\right)\left|\left\langle\varepsilon l^{\prime}\left|\exp \left(i q \cdot r^{\prime}\right)\right| n_{1} l\right\rangle\right|^{2}
$$

where $q$ is the wave vector, $\gamma=1 / \sqrt{\left(1-v^{2} / c^{2}\right)}$ is the relativistic correction and $a_{0}$ is the Bohr radius. This expression can be rewritten in terms of the Generalised Oscillator Strength as

$$
\frac{\mathrm{d}^{2} \sigma}{\mathrm{d} E \mathrm{~d} \Omega}=\frac{4 \gamma^{2} R}{E_{n_{1}} q^{2}} f_{n_{1}}(q)
$$

where the Generalised Oscillator Strength $\mathrm{d} f(E, q) / \mathrm{d} E$ is defined as

$$
f_{n_{1}}(q)=\frac{E_{n_{1}}}{R} \frac{\left|\left\langle\varepsilon l^{\prime}\left|\exp \left(i q \cdot r^{\prime}\right)\right| n_{1} l\right\rangle\right|^{2}}{\left(q a_{0}\right)^{2}}
$$

where $E_{n_{1}}$ is the energy of the excitation and $R$ denotes a Rydberg.

The square of the matrix elements can be expressed in terms of matrix elements involving only radial integrals.

$$
\left|\left\langle\varepsilon l^{\prime}|\exp (i q \cdot r)| n_{1} l\right\rangle\right|^{2}=\left(2 l^{\prime}+1\right) \sum_{\lambda}(2 \lambda+1)\left|\left(\begin{array}{ccc}
l^{\prime} & \lambda & l \\
0 & 0 & 0
\end{array}\right)\right|^{2}\left[\int_{0}^{\infty} \Phi_{n_{1} l}(r) j_{\lambda}(q r) \Phi_{\varepsilon l^{\prime}}(r) d \mathrm{r}\right]^{2}
$$

where the square brackets denote Wigner $3 j$ symbols and $j_{\lambda}(q r)$ are spherical Bessel functions. For the white lines only dipole transitions in which $l^{\prime}=l+1$ need be considered. The optical 
oscillator strength can then be used which is the limit of equation (3) as $q$ tends to zero. The differential cross section becomes

$$
\frac{\mathrm{d} \sigma}{\mathrm{d} E}=\frac{1}{3} \frac{(l+1)}{(2 l+1)} \frac{\left(E_{n_{2}}-E_{n_{1}}\right)}{R}\left[\int_{0}^{\infty} \Phi_{n_{1}}(r) r \Phi_{\varepsilon l^{\prime}}(r) \mathrm{d} r\right]^{2}
$$

where the expansion of the Wigner $3 j$ coefficient has been used for $\lambda=1$. To be consistent with earlier calculations the oscillator strengths in equation (5) were evaluated using wavefunctions generated from the Dirac Slater program of Libermann et al. [15]. Calculations were also performed for ionised atoms.

Table I. - a) Oscittator Strengths for 2p-3ä transitions in 1st row transition elements. b) Oscillator Strengths for $2 \mathrm{p}-3 \mathrm{~d}$ transitions in 1 st row transition ions.

\begin{tabular}{|c|c|}
\hline $\mathrm{Sc}$ & 0.11761 \\
\hline $\mathrm{Ti}$ & 0.1388 \\
\hline $\mathrm{V}$ & 0.15486 \\
\hline $\mathrm{Cr}$ & 0.14483 \\
\hline $\mathrm{Mn}$ & 0.14007 \\
\hline $\mathrm{Fe}$ & 0.18707 \\
\hline $\mathrm{Co}$ & 0.19468 \\
\hline $\mathrm{Ni}$ & 0.20123 \\
\hline
\end{tabular}

\begin{tabular}{|l|l|}
\hline $\mathrm{Sc}^{3+}$ & 0.17659 \\
\hline $\mathrm{Ti}^{4+}$ & 0.22226 \\
\hline $\mathrm{Cr}^{3+}$ & 0.20538 \\
\hline $\mathrm{Cr}^{4+}$ & 0.23394 \\
\hline $\mathrm{Mn}^{2+}$ & 0.18623 \\
\hline $\mathrm{Mn}^{3+}$ & 0.21229 \\
\hline $\mathrm{Mn}^{4+}$ & 0.23848 \\
\hline $\mathrm{Va}^{5+}$ & 0.15486 \\
\hline $\mathrm{Fe}^{2+}$ & 0.19415 \\
\hline $\mathrm{Co}^{2+}$ & 0.20063 \\
\hline $\mathrm{Ni}^{2+}$ & 0.20639 \\
\hline
\end{tabular}

\section{Results and discussion.}

The oscillator strengths for the $2 \mathrm{p}-3 \mathrm{~d}$ excitation in the 1 st row transition elements are given in tables Ia and $\mathrm{Ib}$ for neutral and ionised atoms respectively. All oscillator strengths are per electron 


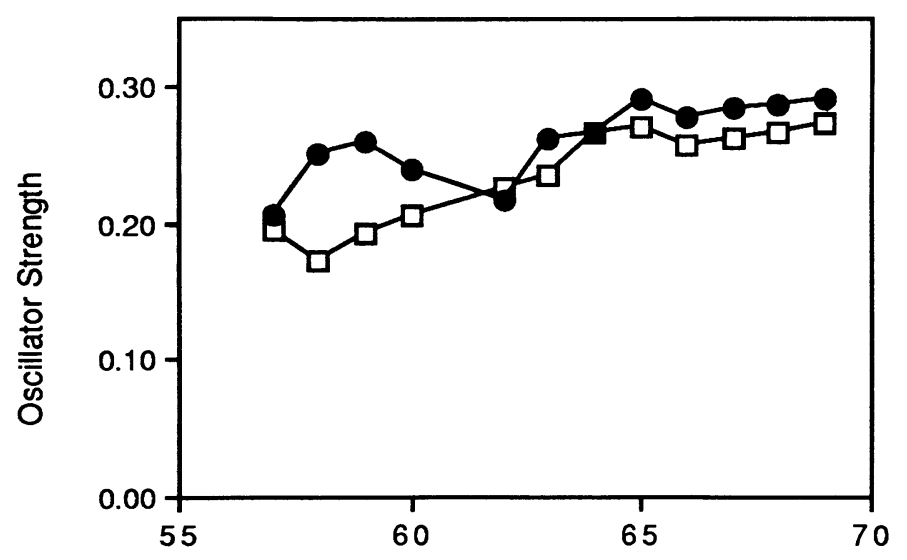

Atomic Number

Fig. 1. - Oscillator strengths for the 1 st row transition elements $2 p-3 d$ transition.

Table II. - Comparison of cross sections for white lines and continuum for 3d transitions elements, assuming $120 \mathrm{kV}, 5.9 \mathrm{mrad}$ collection semi-angle and $100 \mathrm{eV}$ integration window.

\begin{tabular}{|c|c|l|l|c|}
\hline & $\begin{array}{l}\text { Oscillator } \\
\text { Strength } \\
\text { 2p-3d }\end{array}$ & $\begin{array}{l}\text { White Line } \\
\text { Cross Section } \\
\mathrm{cm}^{2}\end{array}$ & $\begin{array}{l}\text { Continuum } \\
\text { Cross Section } \\
\mathrm{cm}^{2}\end{array}$ & $\begin{array}{l}\text { White Line } \\
\text { /Continuum } \\
\%\end{array}$ \\
\hline $\mathrm{Sc}$ & 0.11761 & $2.276 \times 10^{-22}$ & $6.71 \times 10^{-21}$ & 3.4 \\
\hline $\mathrm{Ti}$ & 0.1388 & $2.152 \times 10^{-22}$ & $5.05 \times 10^{-21}$ & 4.26 \\
\hline $\mathrm{V}$ & 0.15486 & $1.924 \times 10^{-22}$ & $3.54 \times 10^{-21}$ & 5.5 \\
\hline $\mathrm{Cr}$ & 0.14483 & $1.445 \times 10^{-22}$ & $2.78 \times 10^{-21}$ & 5.2 \\
\hline $\mathrm{Mn}$ & 0.14007 & $1.127 \times 10^{-22}$ & $1.91 \times 10^{-21}$ & 5.9 \\
\hline $\mathrm{Fe}$ & 0.18707 & $1.222 \times 10^{-22}$ & $1.42 \times 10^{-21}$ & 8.6 \\
\hline $\mathrm{Co}$ & 0.19468 & $1.047 \times 10^{-22}$ & $1.07 \times 10^{-21}$ & 9.8 \\
\hline $\mathrm{Ni}$ & 0.20123 & $0.885 \times 10^{-22}$ & $8.815 \times 10^{-22}$ & 10.0 \\
\hline
\end{tabular}

and should be multiplied by the number of electrons in the shell to obtain an oscillator strength per atom. For comparison with other data it might be necessary to divide by 13.6, the Rydberg unit of energy in $\mathrm{eV}$. The oscillator strength for neutral atoms increases with atomic number as depicted in figure 1. This is because the $d$ electron wave function becomes more compact with increasing atomic number and the matrix element increases in magnitude. For elements lighter than $\mathrm{Mn}$ the oscillator strength in the ionised atom can be $60 \%$ greater than the oscillator strength in the neutral atom, which leads to a constant oscillator strength of about 0.2 across the period. We also calculated the cross sections for excitation to continuum states for the experimental conditions used by Hofer and Golub [16]. In their measurements the accelerating voltage was $120 \mathrm{kV}$, the collection semi angle was $5.9 \mathrm{mrad}$ and an integration window of $100 \mathrm{eV}$ was used. The continuum 


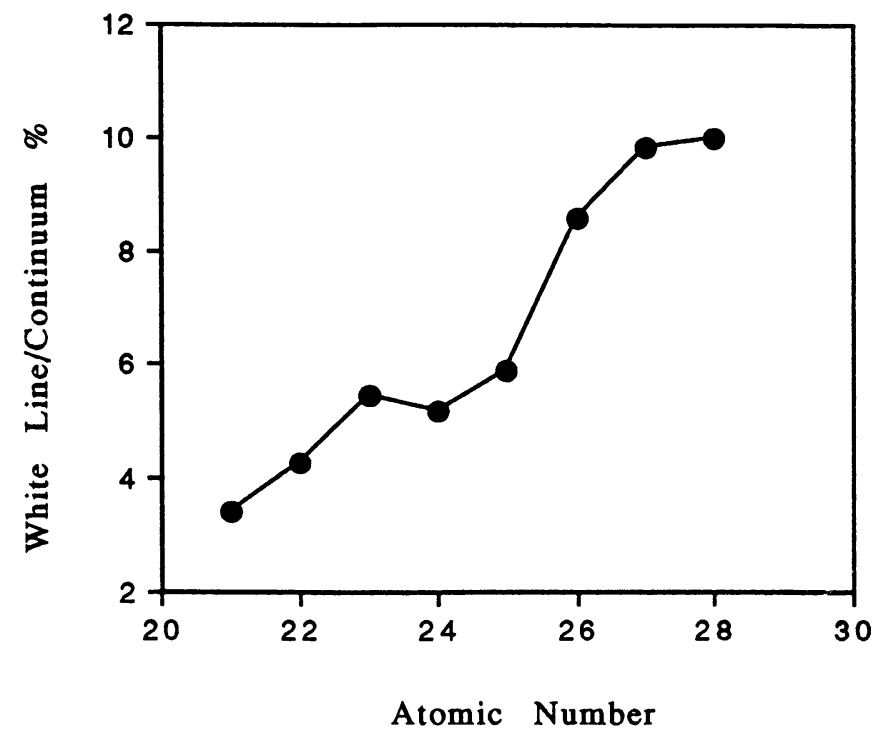

Fig. 2. - Ratio of the white line to continuum intensity for the 1st row transition elements $\mathrm{L}_{23}$ excitation.

Table III. - Oscillator Strengths for $2 \mathrm{p}-4 \mathrm{~d}$ transitions in $2 \mathrm{nd}$ row transition elements and ions.

\begin{tabular}{|c|c|c|c|}
\hline $\mathrm{Y}$ & 0.011285 & $\mathrm{Y}^{3+}$ & 0.018842 \\
\hline $\mathrm{Zr}$ & 0.014845 & $\mathrm{Zr}^{4+}$ & 0.025185 \\
\hline $\mathrm{Nb}$ & 0.017800 & $\mathrm{Nb}^{5+}$ & 0.030895 \\
\hline $\mathrm{Mo}$ & 0.018154 & $\mathrm{Mo}^{6+}$ & 0.036039 \\
\hline $\mathrm{Ru}$ & 0.024700 & $\mathrm{Ru}^{2+}$ & 0.026185 \\
\hline $\mathrm{Rh}$ & 0.026558 & $\mathrm{Rh}^{2+}$ & 0.027881 \\
\hline
\end{tabular}

cross section decreases across the period, due to the energy denominator in equation (2), while the white line cross section slightly increases. The cross section for the white lines and that for the continuum is given in table II and the ratio of white line to continuum intensity is plotted in figure 2 . This ratio increases from about $4 \%$ for scandium to nearly $10 \%$ for nickel. If the white line cross sections for ionised atoms were used, then the ratio of white line to continuum cross section would be 6-7\% rather than $4 \%$ for the lighter elements. These calculated ratios are in agreement with the estimates of Auerhammer et al. [17] using the experimental data of Hofer and Golub [16].

The oscillator strengths for the $2 \mathrm{p}-4 \mathrm{~d}$ transitions in the 2 nd row transition elements are given in table III and plotted as figure 3. Again the neutral atom oscillator strengths show a steady increase with atomic number and the ionised atom oscillator strengths are greater than the values for neutral atoms, sometimes by as much as $70 \%$ to $80 \%$. A spectrum generated from the white line oscillator strength for $\mathrm{Nb}$ and the appropriate continuum oscillator strengths published previously is shown as figure 4. The calculation is compared with the experimental result of Ahn 


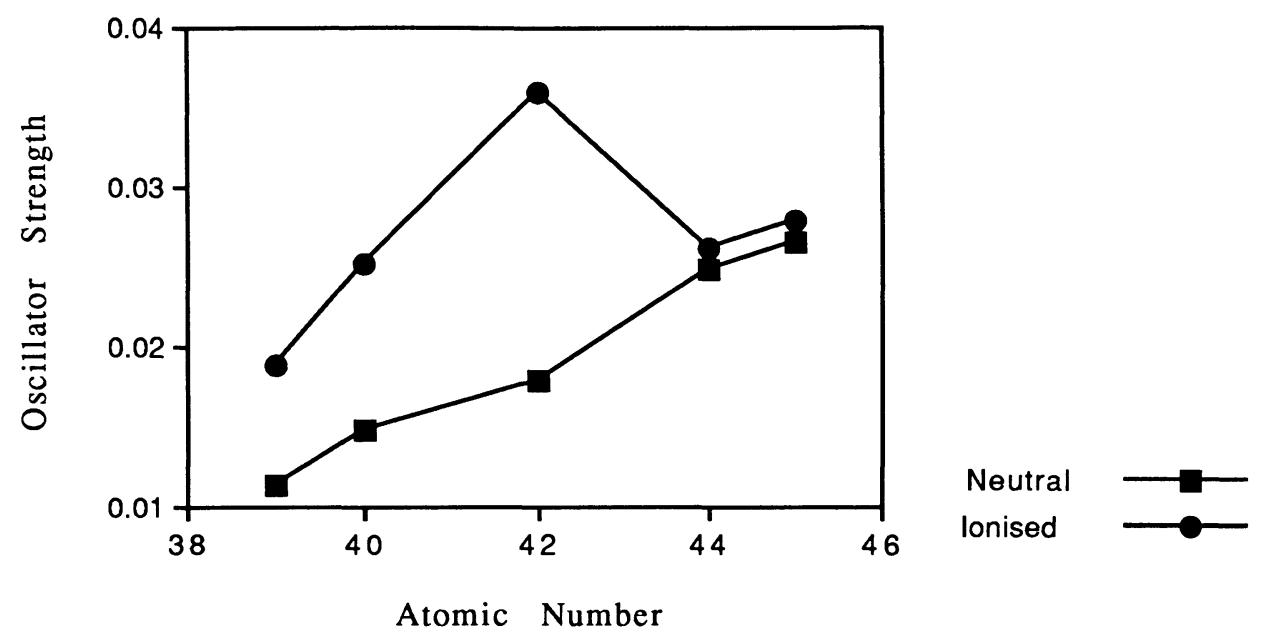

Fig. 3. - Oscillator strengths for the 2 nd row transition elements $2 p-4 d$ excitation. The squares denote neutral atoms and the circles denote ionised atoms.

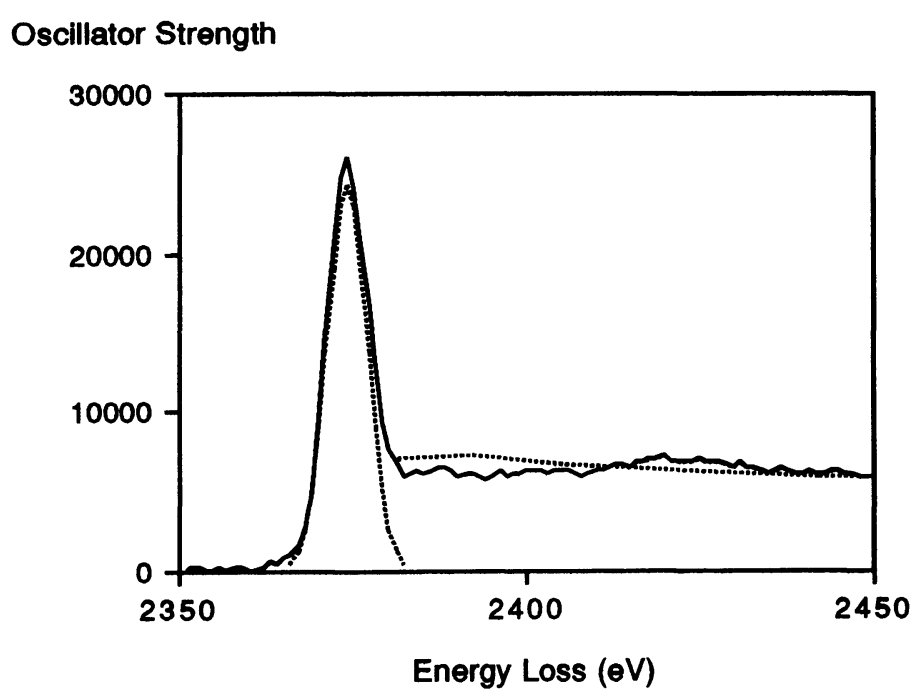

Fig. 4. - Calculated spectrum for $\mathrm{Nb} \mathrm{L}_{3}$ line for $120 \mathrm{kV}, 15 \mathrm{mrad}$ collection angle (dotted line) compared with the experimental result of Ahn and Krivanek (solid line).

and Krivanek shown in the EELS atlas [18] and there is good agreement between calculated and experimental spectra, indicating that the continuum and bound state calculations are in the right proportion.

The oscillator strengths for the $3 \mathrm{~d}-4 \mathrm{f}$ transitions in the rare earth elements for both neutral and ionised atoms are given in table IV. and plotted as figure 5. Although the variation across the period is less than for the $L_{23}$ excitations, there is again a tendency for the oscillator strength for the lighter ionised atoms to be greater than the corresponding oscillator strength for the neutral 
Table IV. - Oscillator Strengths for 3d-4f transitions in rare earth elements and ions.

\begin{tabular}{|l|l|l|l|}
\hline $\mathrm{La}$ & 0.19583 & $\mathrm{La}^{3+}$ & 0.20543 \\
\hline $\mathrm{Ce}$ & 0.17366 & $\mathrm{Ce}^{4+}$ & 0.25094 \\
\hline $\mathrm{Pr}$ & 0.19239 & $\mathrm{Pr}^{3+}$ & 0.25970 \\
\hline $\mathrm{Nd}$ & 0.20627 & $\mathrm{Nd}^{3+}$ & 0.23972 \\
\hline $\mathrm{Pm}$ & 0.21764 & $\mathrm{Pm}^{3+}$ & 0.24812 \\
\hline $\mathrm{Sm}$ & 0.22745 & $\mathrm{Sm}^{3+}$ & 0.21848 \\
\hline $\mathrm{Eu}$ & 0.23632 & $\mathrm{Eu}^{3+}$ & 0.26216 \\
\hline $\mathrm{Gd}$ & 0.26552 & $\mathrm{Gd}^{3+}$ & 0.26839 \\
\hline $\mathrm{Tb}$ & 0.27149 & $\mathrm{~Tb}^{3+}$ & 0.29198 \\
\hline $\mathrm{Dy}$ & 0.25750 & $\mathrm{Dy}^{3+}$ & 0.27913 \\
\hline $\mathrm{Ho}$ & 0.26320 & $\mathrm{Ho}^{3+}$ & 0.28386 \\
\hline $\mathrm{Er}$ & 0.26841 & $\mathrm{Er}^{3+}$ & 0.28810 \\
\hline $\mathrm{Tm}$ & 0.27310 & $\mathrm{Tm}^{3+}$ & 0.29198 \\
\hline
\end{tabular}

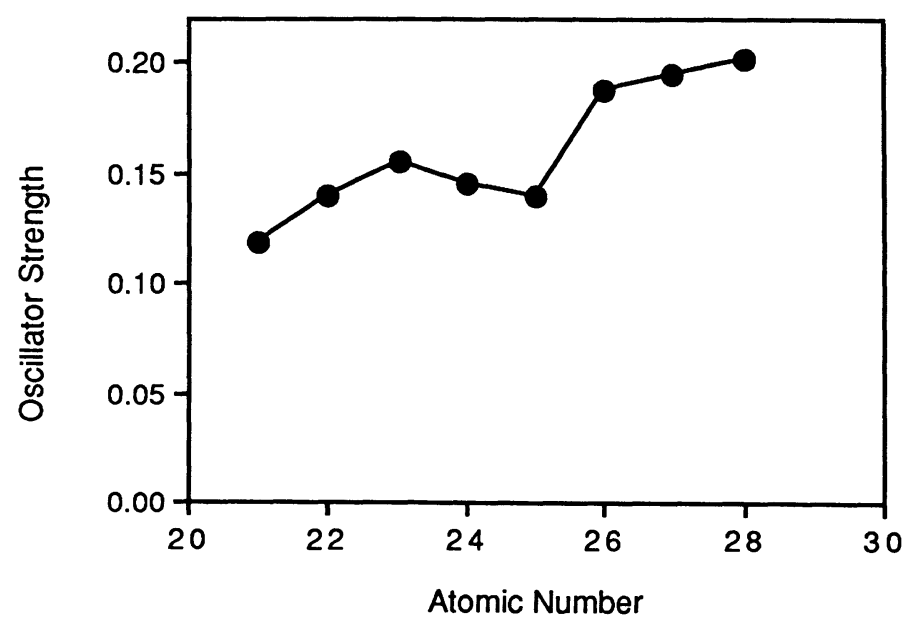

Fig. 5. - Oscillator strengths for the rare earth $3 \mathrm{~d}-4 \mathrm{f}$ excitation. The open squares are for neutral atoms, the filled circles are for ionised atoms.

atoms. Cross sections calculated from these oscillator strengths are given in table V. The experimental conditions of Hofer [19] were assumed, which were the same as the conditions for the measurement of the $\mathrm{L}_{23}$ cross sections mentioned above. The ratio of the white line to continuum intensity is also plotted as figure 6 and shows a steady increase across the period from about $10 \%$ to almost $25 \%$. Again, if ionised white line cross sections were used, the ratio for elements such 
Table V. - Comparison of cross sections for white lines and continuum for rare earth elements, assuming $120 \mathrm{kV}, 5.9 \mathrm{mrad}$ collection semi-angle and $100 \mathrm{eV}$ integration window.

\begin{tabular}{|c|c|c|c|c|}
\hline La & $\begin{array}{l}\text { Oscillator } \\
\text { Strength } \\
\text { 3d-4f }\end{array}$ & $\begin{array}{l}\text { White Line } \\
\text { Cross Section } \\
\mathrm{cm}^{2}\end{array}$ & $\begin{array}{l}\text { Continuum } \\
\text { Cross Section } \\
\mathrm{cm}^{2}\end{array}$ & $\begin{array}{l}\text { White Line } \\
\text { /continuum } \\
\%\end{array}$ \\
\hline $\mathrm{La}$ & 0.19583 & $1.542 \times 10^{-22}$ & $1.776 \times 10^{-21}$ & 8.7 \\
\hline $\mathrm{Ce}$ & 0.17366 & $1.198 \times 10^{-22}$ & $1.604 \times 10^{-21}$ & 7.5 \\
\hline $\mathrm{Pr}$ & 0.19239 & $1.182 \times 10^{-22}$ & $1.347 \times 10^{-21}$ & 8.8 \\
\hline $\mathrm{Nd}$ & 0.20627 & $1.134 \times 10^{-22}$ & $1.116 \times 10^{-21}$ & 10.2 \\
\hline $\mathrm{Sm}$ & 0.22745 & $9.92 \times 10^{-23}$ & $7.735 \times 10^{-23}$ & 12.8 \\
\hline $\mathrm{Eu}$ & 0.23632 & $9.26 \times 10^{-23}$ & $6.450 \times 10^{-22}$ & 14.4 \\
\hline $\mathrm{Gd}$ & 0.26552 & $9.29 \times 10^{-23}$ & $4.959 \times 10^{-22}$ & 18.7 \\
\hline $\mathrm{Tb}$ & 0.27149 & $8.49 \times 10^{-23}$ & $4.178 \times 10^{-22}$ & 20.3 \\
\hline $\mathrm{Dy}$ & 0.25750 & $7.28 \times 10^{-23}$ & $3.850 \times 10^{-22}$ & 18.9 \\
\hline $\mathrm{Ho}$ & 0.26320 & $6.69 \times 10^{-23}$ & $3.253 \times 10^{-22}$ & 20.6 \\
\hline $\mathrm{Er}$ & 0.26841 & $6.14 \times 10^{-23}$ & $2.735 \times 10^{-22}$ & 22.4 \\
\hline $\mathrm{Tm}$ & 0.27310 & $5.63 \times 10^{-23}$ & $2.319 \times 10^{-22}$ & 24.3 \\
\hline
\end{tabular}

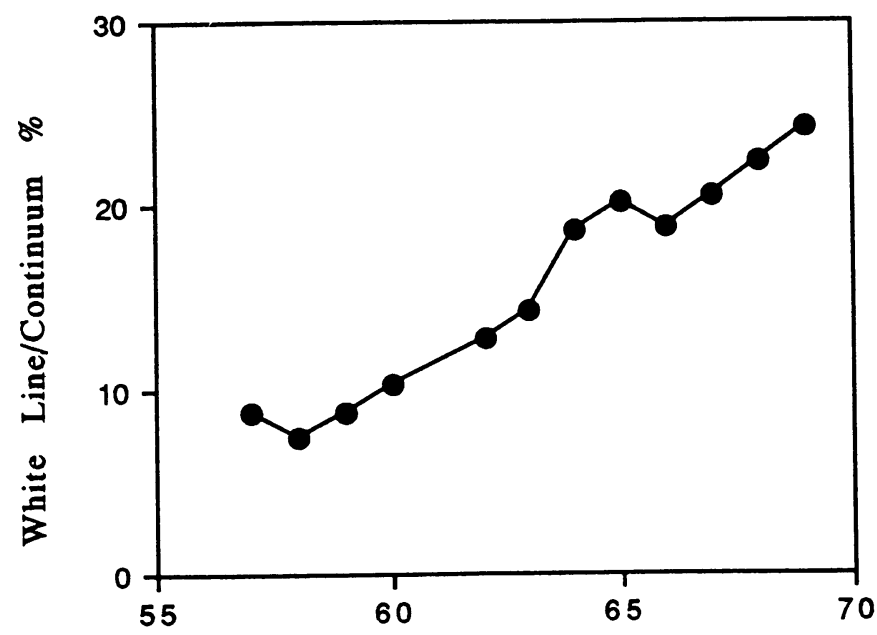

Atomic Number

Fig. 6. - Ratio of the white line to continuum intensity for the rare earth $M_{45}$ excitation. 
as cerium and praesodynium would be closer to $15 \%$. The trend in the calculated ratios is the same as that given by Auerhammer et al. [17] who used the experimental data of Hofer [19] and the EELS atlas, though the Auerhammer et al give a higher estimate of the white line/continuum ratio.

\section{Conclusions.}

We have presented oscillator strengths for the $2 \mathrm{p}-3 \mathrm{~d}$ transitions in the 1 st row transition elements, the $2 p-4 d$ transitions in the 2 nd row transitions elements and the $3 d-4 f$ transitions in the rare earths. These can be used to calculate cross sections for the white line component of the transition metal $\mathrm{L}_{23}$ edges and the rare earth $\mathrm{M}_{45}$ edges.

The oscillator strengths for ionised elements are greater than the neutral atom oscillator strengths, particularly for elements at the beginning of the period. The tabulated oscillator strengths have also been used to calculate the proportion of spectral intensity in the white lines as compared to a $100 \mathrm{eV}$ window in the continuum, assuming a collection semi-angle of 5.9 mrads and $a$ accelerating voltage of $120 \mathrm{kV}$. The white line accounts for up to $10 \%$ of the intensity in the first row transition elements and up to $25 \%$ of the intensity in the $\mathrm{M}_{45}$ edges in the rare earths. These findings are in agreement with the estimates of Auerhammer et al. [17] from the experimental data of Hofer $[16,19]$.

\section{Acknowledgements.}

It is a pleasure to thank Prof Harald Rose and Dr. Helmut Kohl for their hospitality while visiting T.U. Darmstadt and Peter Schorsch for many useful discussions. This work was completed at the Cavendish Laboratory, Cambridge and we would like to thank Profs. A. Howie and L.M. Brown for making their facilities available. We are also grateful to Prof. Ray Egerton for his useful comments.

\section{References}

[1] EgERTON R.F., Electron Energy Loss Spectroscopy in the Electron Microscope (Plenum, New York, 1986).

[2] EGERTON R.F., Ultramicroscopy 4 (1979) 169.

[3] LEAPMAN R.D., REZ P. and MAYERS D.F., J. Chem. Phys. 72 (1980) 1232.

[4] AHN C. and REZ P., Ultramicroscopy 17 (1985) 105.

[5] WENG X. and REZ P., Ultramicroscopy 25 (1988) 345.

[6] LEAPMAN R.D. and GRUNES L.A., Phys. Rev. Lett. 45 (1980) 397.

[7] Yamaguchi T., ShibuYa T., Suga S., and Shin S., J. Phys. C. 15 (1982) 2641.

[8] VAN DER LAAN G. and KiRKMAN I.W., J. Phys. Condens. Matter 4 (1992) 4189.

[9] ThOle B.T. and VAN DER LAAN G., Phys. Rev. B 38 (1988) 3158.

[10] ThOlE B.T., van DER LAAN G., FUgGle J.C., SAWATZKY G.A., KaRNATAK R.C. and EsteVA J.-M., Phys. Rev. B 32 (1985) 5107.

[11] WAdDINGTON W.G., REZ P., GRANT I.P. and HUMPHREYS C.J., Phys. Rev. B 34 (1986) 1467.

[12] Pearson D.H., Fu ltz, B. and AHn C.C., Appl Phys. Lett. 53 (1988) 1405.

[13] MANSON S.T. and KENNEDY D.J., Atom Nuch Data 14 (1974) 111.

[14] SCOFIELD J.H., Atom. Nucl. Data 14 (1974) 121.

[15] Liberman D.A., Cromer D.T. and Waber J.T., Comp. Phys. Commun. 2 (1971) 107. 
[16 HOFER F. and GOLOB P., Micron. and Micr. Acta 19 (1988) 73.

[17] AUERHAMMER J., REZ P. and HOFER F., Ultramicroscopy 30 (1989) 365.

[18] AHN C.C. and KRIVANEK O.L., EELS Atlas (Gatan, Inc. and ASU, Pleasanton, CA, 1983).

[19] HOFER F., GOLOB P. and BRUNEGER A., Ultramicroscopy 25 (1988) 81. 